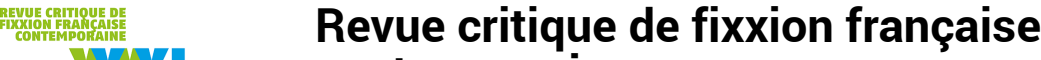 \\ XXI contemporaine
}

Fictions et pouvoirs

\section{Pouvoir et impuissance dans Rosie Carpe de Marie Ndiaye}

Cécile Chatelet

\section{OpenEdition}

1 Journals

Édition électronique

URL : https://journals.openedition.org/fixxion/434

DOI : $10.4000 /$ fixxion.434

ISSN : 2295-9106

Éditeur

Ghent University

Référence électronique

Cécile Chatelet, «Pouvoir et impuissance dans Rosie Carpe de Marie Ndiaye », Revue critique de fixxion française contemporaine [En ligne], 21 | 2020, mis en ligne le 15 décembre 2020, consulté le 17 février 2022. URL : http://journals.openedition.org/fixxion/434 ; DOI : https://doi.org/10.4000/fixxion.434

Ce document a été généré automatiquement le 17 février 2022.

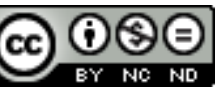

Les contenus de la Revue critique de fixxion française contemporaine sont mis à disposition selon les termes de la licence Creative Commons Attribution - Pas d'Utilisation Commerciale - Pas de Modification 4.0 International 


\title{
Pouvoir et impuissance dans Rosie Carpe de Marie Ndiaye
}

\author{
Cécile Chatelet
}

Rosie se sentait influencée, captive. $(R C, 101)$

1 Dans La sorcière, le personnage principal de Marie NDiaye se désole d'être une magicienne dotée de si faibles pouvoirs : cette sorcière à la vie banale, mère de famille dans un pavillon de banlieue, échoue dans tout ce qu'elle entreprend. De la même manière, le personnage éponyme de Rosie Carpe ${ }^{1}$, paru en 2001, se sent "influencée, captive", et la formule dit tout à la fois l'absence de pouvoir sur sa propre existence et sa difficulté à comprendre ce qui agit sur elle. L'ensemble des romans de l'autrice semblent ainsi aborder la question du pouvoir sous l'angle de l'impuissance : pour les protagonistes, le pouvoir en tant que capacité à agir est quelque chose qui manque. Les différents canevas narratifs sont marqués par l'échec ou la réussite fallacieuse: ils interrogent ce qui fonde la possibilité ou l'impossibilité d'agir des individus.

2 Le pouvoir se perçoit ainsi, dans les textes de Marie NDiaye, depuis le point de vue de celles et ceux qui le subissent. L'autrice s'inscrit en cela - comme de nombreux autres textes de littérature contemporaine, des romans de François Bon à ceux de Virginie Despentes, en passant par le récent Cora dans la spirale de Vincent Message - dans une représentation du pouvoir marquée par une conception relationnelle de ce dernier, à la suite des travaux de Michel Foucault. Rompant avec un pouvoir envisagé comme la caractéristique de certains êtres ou institutions, et avec une pensée juridique du pouvoir et de l'état, il s'agit d'envisager des "situations stratégiques" de pouvoir indissociables de toute forme de relation sociale, et donc de le penser à la fois dans son omniprésence et dans sa possible réversibilité. Ce paradigme d'un pouvoir sans tyran, protéiforme et multidirectionnel, est un défi représentationnel pour la littérature. Dépasser les conceptualisations du pouvoir qui en font l'attribut d'une instance ou d'une personne et passe par une mise en récit qui repose sur l'incarnation donne lieu à de multiples stratégies narratives, la première étant de montrer le pouvoir en creux, par la mise en scène de l'assujettissement de certains protagonistes. Les héros de Marie NDiaye, et ceux de Rosie Carpe en particulier, sont des personnages qui subissent un 
pouvoir souvent sans visage ; leur oppresseur change, il n'a pas toujours conscience d'en être un - à moins que ce ne soit une norme intériorisée qui asservisse, restituée par le biais du discours intérieur. En donnant à voir des formes du pouvoir qui excédent la figure du despote et qui innervent toutes les relations sociales, l'autrice montre l'assujettissement à des principes ou à des injonctions intériorisées.

Si Marie NDiaye creuse le sillon d'une représentation relationnelle du pouvoir, cette lectrice de William Faulkner ${ }^{3}$ met en lumière la difficulté à nommer - et à transformer les rapports de force qui structurent les relations sociales : cette manière de penser le pouvoir souligne ce qu'il a aussi d'invisible ou de dénié. Là où d'autres romans partent de cette omniprésence du pouvoir pour organiser une dénonciation ou mettre au jour un excès, Marie NDiaye prend une autre voie: elle perpétue jusqu'au bout, dans son texte, la logique du pouvoir, en reproduisant la difficulté à le reconnaitre comme tel, et à y résister. Elle l'inscrit également dans un processus historique, et montre la violence d'un ordre social qui empêche de penser des dominations anciennes comme des relations de pouvoir toujours opérantes. Dans Rosie Carpe comme dans les romans faulknériens, le passé informe le présent, et l'héritage de la violence et de la domination d'autrefois structure encore la société représentée - notamment les dominations blanche et masculine. Cet héritage est perceptible tant au niveau individuel, intime, qu'à l'échelle des représentations et des relations sociales : dans Rosie Carpe, Titi, l'enfant de Rosie, porte les stigmates de sa conception, marquée par la violence, l'argent et la honte, tandis que la Guadeloupe dans laquelle la mère et son fils débarquent est toujours imprégnée de colonialisme. L'épaisseur des relations familiales et les dettes héritées ${ }^{4}$ des parents se doublent d'une profondeur historique qui permet de saisir d'un bout à l'autre les mécanismes du pouvoir: des dominations léguées, souvent niées, et la violence d'un ordre social qui se perpétue sans possibilité de révolte.

\section{L'intertexte faulknérien : séparation et exclusion}

La proximité entre l'œuvre de Marie NDiaye et celle de l'écrivain américain a déjà été remarquée : Dominique Rabaté pointait ainsi "l'allure faulknérienne" 5 de Rosie Carpe, soulignant notamment le traitement particulier de la temporalité dans le roman, tandis que Chloé Brendlé mettait en avant la manière commune aux deux auteurs de "rendre le chaos de la psyché de ses héros"' grâce à une maîtrise de la régie narrative qui permet un tressage serré des monologues intérieurs. De manière générale, chez l'autrice, l'entrelacement des thèmes de l'identité, de la famille, du poids de l'histoire et du racisme évoque déjà les romans de William Faulkner. Le travail de la prose de NDiaye rappelle également le style de l'écrivain, ce faulknerese " "fait de digressions et d'accumulations qui mettent à mal la mesure ordinaire de la phrase et imposent des contorsions excessives à la temporalité"8. Au-delà de ces rapprochements généraux, Rosie Carpe semble être un hommage plus direct au septième roman de Faulkner, Lumière d'août. Si le nom de la protagoniste paraît faire signe vers la Rosa d'Absalon, Absalon!, l'héroïne de Marie NDiaye rappelle plutôt Lena Grove, cette jeune fille enceinte qui effectue un long voyage durant tout le mois d'août, pour rejoindre son amant qui ne l'attend pas vraiment. De même, Rosie, enceinte (et déjà affublée d'un premier enfant), va elle aussi jusqu'en Guadeloupe pour retrouver un frère qui ne veut pas réellement l'aider, et elle est, tout comme Lena, constamment associée à la figure 
de l'Immaculée Conception', alors que les deux romans procèdent à de nombreuses reprises à une réécriture des Évangiles. La structure même du roman, partagée entre le point de vue de Rosie et celui de Lagrand, contenant également une longue analepse autour de l'enfance et l'entrée dans l'âge adulte de Rosie, évoque Lumière d'août $t^{10}$. Enfin, la manière dont la couleur jaune nimbe avec insistance l'existence de Rosie ${ }^{11}$, dans le roman de Marie NDiaye, peut aussi se lire comme un écho à la lumière d'août qui accompagne le voyage de Lena et l'ensemble du texte de Faulkner ${ }^{12}$.

5 La réinterprétation discrète mais insistante de ce roman américain de 1932, le premier dans lequel Faulkner aborde de front le racisme ${ }^{13}$, éclaire la manière dont Marie NDiaye aborde, avec Rosie Carpe, la question du pouvoir. Dans le roman de Marie NDiaye comme dans celui de Faulkner, ce sont les valeurs blanches et masculines qui sont au fondement de la loi commune ${ }^{14}$. Ainsi, l'histoire coloniale de la Guadeloupe traverse l'arrivée de Rosie sur l'île, qui rappelle celle des colons français : elle a vêtu son fils d'un "short colonial", tandis qu'elle s'extasie intérieurement de laisser les tracas en métropole, pour trouver, "sur cette terre nouvelle" ( $R C 10)$, la félicité et le succès. Le racisme de la protagoniste est constant, jamais souligné par le texte ni conceptualisé par Rosie elle-même, mais il structure sa psyché : elle a peur, lors de son arrivée à l'aéroport, de ne pas reconnaître son frère Lazare et de le découvrir sous les traits d'un homme noir, qu'elle désigne, au détour d'une pensée, par le terme de nègre:

Titi l'avait vu tout de suite [que ce n'était pas Lazare] et pas elle, tant elle s'était convaincue que Lazare devait avoir changé en cinq ans, qu'il pouvait même avoir revêtu toutes sortes d'aspects inédits. Mais de ne pas le découvrir immédiatement, dès le premier jour, dans la peau d'un nègre, la rassurait tout au fond d'elle. (RC 14)

De la même manière, Rosie refuse d'appeler la Guadeloupe un pays, s'arque-boutant sur l'appartenance administrative de la Guadeloupe à la France, réactivant ainsi la brutalité du passé colonial face à un Noir né sur l'île. Ce lieu n'est "Pas un pays, murmura-t-elle, une région et un département, pas un pays" ( $R C 24)$. Toutes les survivances coloniales et racistes structurant la société française sont ainsi rendues sensibles, sans jamais être évoquées explicitement, de manière conceptuelle ou sous la forme d'une dénonciation. Michel Foucault souligne que la domination est "une structure globale de pouvoir dont on peut trouver parfois les significations et les conséquences jusque dans la trame la plus ténue de la société ; mais c'est en même temps une situation stratégique plus ou moins acquise et solidifiée dans un affrontement à longue portée historique entre des adversaires" ${ }^{15}$. Cette analyse, qui fait d'une des formes du pouvoir une structure pérenne et omniprésente lorsqu'il y a eu cristallisation d'un rapport de force, souligne bien que ce pouvoir est perceptible dans le discours quotidien, dans les clichés qui fondent une pensée, dans les relations sociales ordinaires. La domination des Blancs informe toutes les représentations de Rosie et toutes ses relations aux autres mais, parce qu'elle n'est pas pensée comme telle par les protagonistes qui l'actualisent, elle n'est pas soulignée dans le texte : elle est bien montrée comme un impensé, voire un déni. Ainsi, alors que Lagrand se montre bienveillant mais refuse de mentir à Rosie sur la situation catastrophique $d u$ frère de cette dernière, Rosie, paniquée, songe soudainement :

Mais pourquoi cracher à nos pieds de malheureux Blancs à peine débarqués, se demandait-elle en même temps, et n'ayant causé de tort à personne sur cette terre de Guadeloupe ? (RC 21)

7 L'ironie de l'énoncé est terrible: l'oubli manifeste chez Rosie des violences de la colonisation commises par les Blancs en Guadeloupe et des injustices subies par les 
Noirs pendant des siècles à la suite de l'arrivée des colons confine au déni, et la perpétuation d'une "structure globale de pouvoir" écrasant les populations noires repose aussi sur la dénégation par les Blancs de la situation de domination dans laquelle ils se trouvent.

\section{Un ordre économique impérieux : imaginaire social et argent}

8 Si Rosie fait preuve de racisme, elle est aussi la victime d'une autre domination qui structure la société, le sexisme : celle qui assujettit dans un rapport social peut être dominée dans un autre. Dans le roman, domination masculine et exploitation des femmes vont de pair, l'idéologie capitaliste venant légitimer les inégalités de genre, si celles-ci permettent de gagner de l'argent. André Bleikasten soulignait que les romans de Faulkner montraient bien que les dominations blanches et masculines avaient besoin d'une composante "plus avouée, plus officielle", par laquelle "la communauté définit ses valeurs et légitime ses priorités" ${ }^{" 16}$. Si, chez l'écrivain américain, il s'agit du puritanisme, chez Marie NDiaye cet ordre transcendant qui organise les hiérarchies, c'est le capitalisme. Lorsqu'il analyse l'aliénation individuelle et collective, Cornelius Castoriadis considère qu'une société aliénante, "hétéronome", est une société instituée de sorte que ses normes sociales ou ses représentations du monde soient considérées par les individus comme des valeurs absolument justes et vraies, et non comme des créations qu'elle a elle-même produites. Or, si cette société aliénante est, de manière paradigmatique, religieuse, il souligne que la société contemporaine capitaliste interdit également toute remise en cause concernant ses valeurs et son imaginaire :

Le trait caractéristique du capitalisme entre toutes les formes de vie socialhistoriques est évidemment la position de l'économie - de la production et de la consommation, mais aussi, beaucoup plus, des critères économiques - en lieu central et valeur suprême de la vie sociale [...] toutes les activités humaines et tous leurs effets arrivent, peu ou prou, à être considérés comme des activités et des produits économiques, ou pour le moins, comme essentiellement caractérisés et valorisés par leur dimension économique. ${ }^{17}$

9 L'ordre religieux s'est mué en ordre économique, et il justifie d'utiliser les femmes sans avoir à s'en expliquer : c'est ainsi que Max, le patron de Rosie dans l'hôtel où elle trouve son premier emploi, instrumentalise immédiatement l'héroïne et leurs rapports intimes. Rapidement, les relations sexuelles que Max a avec Rosie ne l'intéressent plus en elles-mêmes, et il laisse une femme filmer leurs ébats en échange d'argent, mettant Rosie devant le fait accompli. Le discours indirect libre permet de retracer la force de la domination qui s'exerce sur cette dernière, sans menace physique, et la confrontation de Rosie à son propre asservissement :

Maintenant Rosie Carpe n'éprouvait plus ni tendresse ni fierté, qu'une pauvre haine impuissante, honteuse, dont elle n'avait plus la moindre gratitude à Max. Rosie savait qu'elle était cette Rosie Carpe-là mieux encore qu'elle n'avait été l'autre, celle qui pourtant plaisait tant [à Max], hardie, satisfaite, confiante et moqueuse. Elle attendait dans son lit, droite, gelée, profondément désorientée. Elle savait que personne ne l'obligeait à accepter cela. Cette femme et sa caméra, même Max, rien ne la forçait à accepter leur entrée dans sa chambre. Elle avait compris que Max faisait payer la séance, et, quand même bien elle eût voulu sa part, elle savait que Max n'avait jamais eu aucune intention de la lui donner. Il prenait l'argent et feignait de considérer qu'ils étaient d'accord, Rosie et lui, pour que cela se passe 
ainsi, que Rosie était contente de cette fantaisie amoureuse, qu'il s'y pliait.

(RC 86-87)

Rosie saisit qu'il est convenu que personne ne lui doit rien: elle peut être instrumentalisée et on nie l'existence même de sa volonté, que Max, dans son esprit, remodèle à sa guise en fonction de ses intérêts. Si l'héroïne pense que "rien le la forç[e] à accepter cela", soulignant l'absence de contrainte physique ou économique directe (tout en éclairant un sentiment de culpabilité de la victime d'une domination), la haine "impuissante" qu'elle ressent met en lumière la conscience diffuse, chez Rosie, du fait que tout ce qu'elle pourrait dire ou faire dans cette situation ne servirait certainement à rien : il est très difficile de s'opposer à un ordre social qui ne lui accorde aucune valeur, qui fait de l'argent la justification ultime et légitime son exploitation. Max a l'audace de dire à Rosie qu'ils ne peuvent pas se parler trop longtemps pendant leur service à l'hôtel : “Après le boulot, c'est mieux. Moi, je ne mélange jamais la baise et le travail” ( $R C$ 80) explique-t-il. L'ironie fonctionne ici à plein. Le personnage reprend un cliché, et la phrase fait sens pour Max au vu du contexte, mais les termes de l'énoncé tiennent aussi de l'antiphrase : le protagoniste fait exactement ce qu'il prétend éviter par professionnalisme et, dans le monde de Rosie Carpe, le sexe est devenu un travail, particulièrement aliénant pour les femmes. Cette ironie provient de la superposition de l'intention satirique du narrateur à la voix d'un personnage inconscient qui, lui, en est dépourvu. On la retrouve dans les propos de la femme qui les filme, lorsqu'elle félicite Rosie et Max : "Les amateurs, les vrais amoureux, ceux qui le font pour le plaisir, c'est ce qui marche le mieux" ( $R C$ 89). La référence à une catégorie de l'industrie pornographique, l'inadéquation entre ce que cette femme tente de montrer dans son film et la situation réelle - au-delà même de l'absence de plaisir pris par Max et de la souffrance de Rosie, la femme demande à cette dernière de pleurer devant la caméra sont brutales, et la phrase s'achève par la réduction cynique du plaisir (ici purement apparent) à sa valeur marchande. Rosie réalise peu à peu la place à laquelle elle est cantonnée dans cette société mercantile qui fait d'elle un objet utile, mais de peu de valeur. Repensant à Max, elle songe : “je lui ai plu aussi parce que son instinct d'homme intéressé, quoique pas méchant, lui a fait comprendre que je ne lui demanderais jamais rien de plus terrible que de se taire un peu" ( $R C$ 93). Le roman ne fait pas de Max une figure diabolique, mais l'incarnation de la bêtise et de l'ère du temps: il utilise le pouvoir que la société lui donne pour tenter de réussir. Lakis Proguidis a déjà souligné que Rosie Carpe pouvait être lu comme un "grand roman du monde abstrait de l'argent"18, dans lequel les personnages sont privés de toute possibilité de désir érotique. Les femmes n'hésitent pas non plus à instrumentaliser d'autres femmes à des fins pécunières. Diane, à nouveau mère des années après avoir eu Lazare et Rosie, vante les mérites de sa dernière fille - dont elle regrette par ailleurs que ça ne soit pas un garçon -, qu'elle prostitue : "elle vaut de l'or" dit-elle ( $R C 392)$, et la remotivation de l'expression figée est ici toute significative.

11 Au-delà de l'assujettissement des femmes, c'est bien l'argent qui dirige et valide les conduites. Lorsque Rosie retrouve sa mère, cette dernière est transformée par ce qu'elle vient de gagner grâce à des placements boursiers :

Ton père a démissionné et j'ai revendu mon cabinet. Nous avions fait des placements judicieux, tu sais, il y a une quinzaine d'années. La Bourse a été bonne pour nous. Elle a fait de nous qui n'étions, au fond, quoi ? Pas grand-chose, Rosie, de tout petits bourgeois - eh bien, des actionnaires prospères. Nous pourrions te conseiller, Rosie. À propos de placements. Nous avons tout compris, ton père et 
moi. La Bourse a été bonne pour nous comme jamais rien ni personne d'autre.

(RC 154) accédé à un nouveau statut, hautement enviable puisqu'il évacue toute idée de travail : ils sont "des actionnaires prospères". Le discours est ambivalent: selon la mère de Rosie, ils ont à la fois mérité leur fortune, puisqu'ils ont eu l'intelligence de faire les bons placements, mais leur métamorphose en rentiers tient aussi du miracle: ils se trouvent couverts d'or comme on est touché par la grâce, et la bourse, parée d'une majuscule, devient ainsi la nouvelle Providence. L'argent justifie tant le comportement de Max avec l'héroïne que les transformations monstrueuses de la mère de Rosie, qui rajeunit au fil du livre ${ }^{19}$; il est la mesure de toute chose.

\section{Rêves de succès et asservissement}

Le roman de Marie NDiaye articule argent, désirs et asservissement : les aspirations des protagonistes sont liés à des narrations du succès, qui ont tout à voir avec les possessions matérielles et la réussite sociale. Ces récits structurent la société et conditionnent la manière dont les personnages lisent leurs existences. Si l'aliénation, selon Cornelius Castoriadis, "c'est la domination par un imaginaire autonomisé qui s'est arrogé la fonction de définir pour le sujet et la réalité et son désiri ${ }^{20}$ ", alors la croyance en l'importance supérieure de l'argent repose sur la circulation de récits qui fondent un imaginaire social commun, et le structurent autour de critères économiques. Ces récits articulent richesse et bonheur, en mettant en scène possessions matérielles, signes extérieurs de succès et réussite dans le domaine du commerce. Devenu la destinée rêvée par chacun, l'argent dirige les conduites des personnages par le biais de récits. On trouve une de ces narrations qui font de l'argent la valeur suprême et guident les personnages dans le discours de Max, que Rosie se remémore :

il se disait libéral, réaliste, ambitieux. Il consultait une voyante qui lui avait confirmé qu'il serait bientôt gérant, puis directeur, enfin gros actionnaire de la chaîne hôtelière qui l'employait et qu'il n'aurait plus un jour qu'à se prélasser en regardant l'argent tomber. (RC 82)

14 Max se raconte un scénario stéréotypé ascendant et prometteur, et la voyante est là pour confirmer la narration que Max s'est choisie: une évolution professionnelle grandiose, dans laquelle on touche au but en étant riche sans plus travailler. Même les personnages moins antipathiques que Max sont pris dans ces récits. Le discours intérieur de Lagrand au sujet de sa voiture, lorsqu'il la conduit, la lie aux images d'une existence idéale :

Son pick-up était parfait - pas un simple véhicule, non, mais la matérialisation d'une atmosphère idéale, l'incarnation de métal, de plastique et de cuir de la grâce absolue et discrète, aux yeux de Lagrand, d'une matinée dominicale en avril, Guadeloupe. Son pick-up était parfait. Il lui avait coûté près de deux cent mille francs et Lagrand en payait chaque mois le remboursement. ( $R C$ 202)

La scansion de la phrase appliquant l'idée de "perfection " à une voiture rappelle bien sûr la publicité ; la voiture, en tant qu'objet de réussite sociale, raconte l'histoire d'un destin heureux lié aux possessions matérielles et à la reconnaissance. La mention explicite du prix élevé rappelle que l'argent est au cœur de l'affaire - on compte tout dans les récits de Marie NDiaye, même ce qu'il est souvent impossible de quantifier - ; il est la voie vers le salut, il constitue en lui-même la seule « grâce » qui apparaît possible 
aux personnages, notamment à ceux qui, comme Lagrand, sont épris de perfection mais qui sont minés par un échec ou une douleur refoulés. Chloé Brendlé a déjà souligné que le recours au discours intérieur chez Marie NDiaye permettait de percevoir les discours et clichés absorbés par les personnages :

Davantage que des fictions de l'intériorité, les romans de Marie NDiaye sont alors des récits de l'intériorisation, au double-sens psychologique et sociologique du terme; ils ne montrent pas tant le processus de la pensée en action que celui de l'incorporation de normes extérieures..$^{21}$

16 Ces discours et ces clichés, qui prennent presque toujours la forme de récits directs ou indirects du succès et de la richesse, constituent des injonctions sociales et sont une des mécaniques concrètes du pouvoir mises en scène dans le livre. Ces narrations, qui formatent les désirs en les faisant correspondre à la norme sociale, traversent tous les personnages du roman, ils sont liés à "une condition sociopolitique et existentielle commune"22, à une hiérarchie sociale partagée qui guide les conduites, qui limite ou contraint impérieusement les protagonistes. Ils rappellent en cela le storytelling tel qu'il est analysé par Christian Salmon, qui les différencie des autres narrations :

Des grands mythes du passé au roman moderne, la fonction des récits a toujours été d'explorer les conditions d'une expérience possible - les nouveaux rapports au corps, au temps et à l'espace - d'inventer comme le disait Deleuze "un peuple qui manque". Les nouveaux récits que nous propose le storytelling n'explorent pas les conditions d'une expérience possible, mais les modalités de son assujettissement. ${ }^{23}$

Il ne reste aux êtres humains qu'à jouer les rôles attendus, qu'à tenter de reproduire mécaniquement, sans désir, des narrations orchestrant la reproduction d'un ordre social qui valorise la seule richesse. Dans une société organisée autour du succès et de l'argent, ceux qui en sont dépourvus sont tenus à l'écart, tout en cherchant désespérément à entrer dans la norme pour trouver leur place dans le monde. Avant que Lazare, le frère de Rosie, ne parte en Guadeloupe, il explique bien qu'il compte s'y rendre pour devenir riche - la destination lui importe peu, il suit simplement son associé Abel et est prêt à vendre n'importe quoi, comme il l'explique à sa sœur :

Maintenant, il s'agit de gagner de l'argent, le plus possible. Avec Abel, je peux le faire. Et après, Rosie ? C'est comme ça. En Guadeloupe, pourquoi pas, puisque c'est l'idée d'Abel qui connaît tout, ou ailleurs, peu m'importe. J'irai où il faut. Que veuxtu que je te dise, Rosie? C'est comme ça. Pas question pour moi de rester sur le carreau, maintenant. Je veux ma part, maintenant, Rosie. Que je ne l'ai pas ne change rien au monde pour personne, alors, Rosie, autant que je l'aie, comme ceci ou comme cela, peu importe. La part de richesse qui me revient, Rosie. Je ferai tout pour l'avoir, maintenant. (RC 167-168)

L'exclusion, cette réalité du fonctionnement social qui laisse "sur le carreau" ceux qui n'arrivent pas à se conformer aux attentes et aux valeurs du groupe, est ici palpable. Il n'y a pas de remise en cause de la norme dans le discours de Lazare, mais un besoin désespéré d'y entrer pour exister : seul le fait de gagner de l'argent importe, "d'avoir sa part", pour être sûr d'en être. Lazare peut tout sacrifier à l'ambition d'être riche, s'il le "faut", tandis que la répétition du "c'est comme ça" souligne que Lazare ne fait que se conformer à la règle du jeu, aux principes sociaux qui lui apparaissent clairement et qui l'ont jusqu'à présent exclu. Mais le destin de Lazare est malheureux, et la success story n'a pas lieu: son entreprise commerciale échoue, il se livre à divers trafics avant de passer quelques années en prison, puis de rentrer dans la ville d'origine de sa famille, Brive-la-Gaillarde - dont le nom résonne alors de manière sarcastique. Lazare n'est arrivé nulle part, ou plutôt, il ressuscite exactement à son point de départ: les 
narrations de la réussite auxquelles il a pourtant essayé de se conformer sont restées des histoires stéréotypées, et sa vie est ainsi jugée ratée par sa mère :

Quel échec, fit-elle, glaciale. Brive-la-Gaillarde : j'ai honte pour lui et presque de lui, monsieur Lagrand. Il ne m'a pas écrit et cela vaut mieux. Mon petit Lazare a tout raté. (RC 391)

Le jugement définitif de la mère sur son fils, excluant ce dernier de sa vie comme il est banni du monde, est d'autant plus intéressant qu'elle est, elle, devenue riche. Danielle change son prénom pour Diane, elle se réinvente sans cesse dans différentes narrations du succès, tantôt actionnaire, tantôt femme au foyer idéale à la limite de l'égérie pour magazines de décoration, et même gérante de restaurant, changeant de mari pour être plus en accord avec sa nouvelle existence. La débine ou la réussite, l'échec ou le succès : l'opposition structure tout le roman comme elle structure la psyché des personnages. Pour les individus maintenus hors des récits du succès, il ne reste que le néant. Tout comme son frère, Rosie a le sentiment de rester à côté des récits de la société, et de l'histoire elle-même. Exclue de tout, elle doute de sa capacité, à elle et à son fils Titi, d'exister en tant qu'individus :

Mais elle sentait bien que toute sa chair chaude et moite se figeait à nouveau dans l'impression éprouvée durant longtemps, temporairement chassée (car la Guadeloupe, la Guadeloupe ! se disait-elle depuis des mois, rêvant d'un rêve poudré d'or), de se mouvoir, elle et Titi, à côté du récit, en dehors d'une vaste et complexe histoire que les autres, même les moins bien lotis, vivaient activement. Rosie et Titi, lui semblait-il, n'étaient tout simplement pas là, sans que leur absence fût même signalée par deux ombres ou deux silhouettes fantomatiques, et Rosie pensait savoir maintenant que leurs rôles n'avaient été prévus par personne, qu'ils ne pouvaient, elle et Titi, qu'entrer en force dans le cours d'existences qui coulait sans eux et sans nul besoin d'eux. (RC 33 ; je souligne)

Rosie ne dit pas directement qu'elle est en marge de la société, mais elle se sent tellement "en dehors" de l'histoire de la communauté qu'elle ne se sent plus exister: les différences entre les narrations du succès et le récit qu'elle peut se faire de sa propre vie sont trop patentes, son existence est non-conforme. Aucun "rôle" social n'existe pour ceux qui ne sont pas touchés par la grâce de l'argent et du succès.

\section{Conclusion}

21 Pour représenter le pouvoir, Marie NDiaye ne passe pas par le mode de la dénonciation: son roman rejoue le fonctionnement des récits sociaux à travers le discours intérieur des personnages. Cette recréation minutieuse d'un pouvoir qui configure les relations sociales comme les stratégies existentielles montre des protagonistes asservis par l'argent, pris dans des dominations héritées. L'œuvre de Marie NDiaye met en lumière donc une difficulté à résister: c'est l'impuissance des protagonistes à s'élever contre ces formes de pouvoir qui permet de les représenter, à l'inverse de nombre de fictions contemporaines qui choisissent de mettre en scène des soulèvements contre le pouvoir (même si ce sont souvent leurs échecs qui sont narrés). C'est peut-être dans la forme même du récit de l'autrice qu'on trouve l'opposition la plus nette aux pouvoirs mis en scène. Le roman est fait de multiples épisodes qui s'interrompent souvent de manière brutale, et il refuse toute narration téléologique. Sa construction s'oppose ainsi frontalement aux récits de l'argent et du succès qui dominent la société et la psyché des personnages, qui sont, eux, ascendants et linéaires. 
Si Rosie se sent hors des narrations qui fondent notre imaginaire social, elle est le personnage principal du récit de Marie NDiaye: dans ce cadre narratif-là, elle est un objet de récit légitime, un sujet focalisant pour l'autrice, et elle existe.

\section{NOTES DE FIN}

1. Marie NDiaye, Rosie Carpe, Paris, éditions de Minuit, 2009 [2001], dorénavant RC.

2. “[...] le pouvoir ce n'est pas une institution et ce n'est pas une structure, ce n'est pas une certaine puissance dont certains seraient dotés : c'est le nom qu'on prête à une situation stratégique complexe dans une société." Michel Foucault, Histoire de la sexualité. La volonté de savoir, Paris, Gallimard, 1976, <Tel>, p. 123.

3. "Faulkner est le seul écrivain, je crois, que j'ai relu et relu." Marie NDiaye : l'étrangeté à l'œuvre (dir. Andrew Asibong et Shirley Jordan), "Rencontre avec Marie NDiaye", Lille, presses universitaires du Septentrion, <Revue des Sciences Humaines>, p. 191.

4. Dans Rosie Carpe, Titi hérite de toutes les violences dont sa mère, exploitée sexuellement et abandonnée, a été victime et, si Rosie ne peut les nommer, elle connaît le poids de son passé et ses conséquences sur son enfant: "On ne pouvait pas faire que ce qui s'était passé et la manière dont cela avait eu lieu se soit passé différemment. Si ce qu'il y avait en [Rosie] devait voir le jour, il porterait fatalement le signe qu'on s'était payé sur lui, que tout un tas de gens allaient se payer sur lui pendant longtemps encore." (RC 96)

5. "comme si le temps obéissait à deux régimes distincts : celui d'une durée lente, immobilisée, frappée de stupeur (c'est le temps des pensées de Rosie, puis dans la troisième partie, à l'occasion d'un extraordinaire relais narratif, celui des pensées de Lagrand) qui s'oppose au temps de la métamorphose fantastique, aux changements de personnalité (et c'est alors le temps sur lequel règne Danielle Carpe, celle qui, au lieu de vieillir comme son inconsistant mari, rajeunit et se transforme volontairement en Diane, déesse d'une nouvelle sexualité mercantile)" Dominique Rabaté, Petite physique du roman, Paris, José Corti, 2019, <Les Essais>, p. 211.

6. Chloé Brendlé, "Le discord intérieur des personnages de Marie NDiaye", Revue critique de fixxion française contemporaine, $\mathrm{n}^{\circ} 3$ "Fictions de l'intériorité" (dir. Alexandre Gefen \& Dominique Rabaté), 2016 [en ligne]

7. Ce terme désigne de manière humoristique les particularités de l'écriture de Faulkner, que Philip M. Weinstein décrit ainsi: "Faulknerese is a verbal practice committed to proliferated syntax and Latinate/polysyllabic vocabuary. Its insistence manifests itself in sentence after sentence that thunders onward. "Faulknerese " does not select or pare down, and it has little interest in the single, telling detail or in pausing and letting the reader catch his breath." Philip M. Weinstein, Simply Faulkner, New York, Simply Charly, 2016, p. 75.

8. Gabrielle Adjerad, Le jeu du pouvoir. Grotesque et liberté dans Absalom, Absalom ! et Light in August de William Faulkner, mémoire de master 2 soutenu à l'université Paris IV - Sorbonne sous la direction de Marc Amfreville, septembre 2013.

9. Dans le roman de Marie NDiaye, on trouve par exemple "J'ignore ce qui m'a rendue enceinte, dit Rosie. [...] Quelque chose s'est passé à quoi je n'ai pas assisté, et après j'étais enceinte” ( $R C 21)$, tandis qu'André Bleikasten écrit, à propos de Lena: "Déesse toute ensemble lumineuse et terrestre, grecque et chrétienne, vierge et mère. On a beau savoir que Lena a cédé aux avances d'un gredin, tout semble indiquer que l'identité du géniteur est pour elle sans importance et qu'il 
n'y a donc aucune urgence à le retrouver. Lena est celle à qui il ne manque rien et qui nous manque à jamais : la Mère d'avant la séparation, antérieure à la naissance et à la mort, en amont du temps. Comblée et intacte : mère et vierge." André Bleikasten, "Notice, notes et variantes", in William Faulkner, Oeuvres romanesques, Paris, Gallimard, <Bibliothèque de la Pléiade>, 1995, p. 1139.

10. Le roman de Faulkner se construit lui aussi sur les points de vue de Lena Grove et Joe Christmas, laissant une large place à une analepse revenant sur la vie de ce dernier, qui évoque, comme pour Rosie, son enfance et ses terribles premières amours.

11. L'omniprésence de la couleur jaune a été largement remarquée : Sarah Burnautzki la lie à l'incorporation de l'expérience de la violence et de l'abus (Sarah Burnautzki, "Effets d'épouvante dans Rosie Carpe et Rosemary's Baby", L'Esprit Créateur, vol. 53, n², "Marie NDiaye's Worlds / Mondes de Maria NDiaye", Baltimore, John Hopkins University Press, 2013, p. 42-55), tandis que Maud Fourton souligne astucieusement que ce jaune fait tout à la fois signe vers l'or des dieux, et le soufre de Lucifer (Maud Fourton, "Marie NDiaye, Rosie Carpe : une fiction du tant bien que mal au malgré tout, une fiction de l'ambivalence et de la complicité", Christian Oster et Cie: retour du romanesque (dir. Aline Mura-Brunel), Amsterdam/New York, Rodopi, 2006, p. 49-63).

12. Dans le texte de Faulkner, on trouve par exemple ce passage : "It seemed to him that he could see the yellow day opening peacefully on before him, like a corridor, an arras, into a still chiaroscuro without urgency. It seemed to him that as he sat there the yellow day contemplated him drowsily like a prone and somnolent yellow cat" William Faulkner, Light in August, New York, Vintage international, 1990 [1932], p. 111-112.

13. Comme le souligne André Bleikasten dans son introduction à l'œuvre de William Faulkner, op. cit. p. 1144.

14. "Valeurs masculines, valeurs blanches: elles sont au principe de la Loi commune et s'imposent à l'ensemble du corps social comme à l'individu, et à l'individu intégré autant qu'au marginal ou au déviant. Malheur aux contrevenants : tôt ou tard ils seront démembrés - non seulement ostracisés, mais roués, brisés, corps et âme." André Bleikasten, "Notice, notes et variantes", op. cit. p. 1147-11148.

15. Michel Foucault, "Le sujet et le pouvoir", Dits et écrits. Tome II, Paris, Gallimard, 1982,<Quarto>, p. 1062.

16. André Bleikasten, op. cit. p. 1148.

17. Cornélius Castoriadis, Les carrefours du labyrinthe, tome 5: Fait et à faire, Paris, Seuil, 2008 [1997], <Points>, p. 169.

18. Lakis Proguidis, "Les enfants de la Bourse", L'atelier du roman, $\mathrm{n}^{\circ} 35$, "Rosie Carpe de Marie NDiaye", Paris, Flammarion, 2003, p. 81.

19. Le rajeunissement de Danielle/Diane rappelle un motif repéré par John T. Irwin chez Faulkner. Le critique s'appuie sur ce que le psychanalyste Ernest Jones a appelé "le fantasme du renversement de générations", qui correspond à une croyance relativement répandue chez les jeunes enfants: ils s'imaginent que lorsqu'ils grandiront, ils deviendront nécessairement les parents tandis que leurs parents redeviendront leurs enfants. Si l'on suit cette logique, alors les enfants en viennent à occuper symboliquement la place des grand-parents. Ce trope semble, chez Marie NDiaye, condenser les dettes familiales héritées, les hantises de l'histoire, la fascination contemporaine pour la jeunesse et l'injonction sociale à la réinvention de soi. John T. Irwin, Doubling and incest/ Repetition and revenge. A Speculative Reading of Faulkner, Baltimore / London, John Hopkins University Press, 1975.

20. Cornelius Castoriadis, L'institution imaginaire de la société, Paris, Seuil, 1999 [1975], <Points >, p. 152.

21. Chloé Brendlé, op. cit.

22. Ibid. 
23. Christian Salmon, Storytelling. La machine à fabriquer des histoires et à formater les esprits, Paris, La Découverte, 2007, p. 199.

\section{RÉSUMÉS}

Dans Rosie Carpe, Marie NDiaye aborde la question du pouvoir sous l'angle de l'impuissance : pour les protagonistes, le pouvoir en tant que capacité à agir est quelque chose qui manque. Le roman représente un pouvoir sans tyran et protéiforme, que l'autrice montre en creux, par la mise en scène de l'assujettissement de certains protagonistes. Un intertexte faulknerien parcourt le livre, mettant en lumière la difficulté à nommer - et à transformer - les rapports de force qui structurent les relations sociales, au sein d'une communauté toujours marquée par la domination blanche et masculine. La dimension subversive du texte de Marie NDiaye ne se fait pas sur le mode de la dénonciation, elle n'est pas liée à la représentation de la révolte, mais à la recréation minutieuse d'un pouvoir omniprésent, qui configure les relations sociales comme les stratégies existentielles.

INDEX

Mots-clés : roman, pouvoir, domination

\section{AUTEURS}

\section{CÉCILE CHATELET}

Paris 3 - Sorbonne Nouvelle 